\title{
The indefeasibility of the inference that if not-A, then not-C
}

\author{
Ingrid Van Canegem-Ardijns * \\ University of Leuven, Dutch, German and Computational Linguistics, Blijde-Inkomststraat 21, Postbus 3308, B-3000 Leuven, Belgium
}

Received 24 November 2008; received in revised form 23 March 2009; accepted 8 May 2009

\begin{abstract}
Content and, to a lesser degree, epistemic or inferential conditionals regularly invite conditional perfection as a non-monotonic inference or conversational implicature. Conditional perfection (henceforth $\mathrm{CP}$ ) is the natural language tendency to perfect conditionals (if A then C) into their corresponding biconditionals (if and only if A, then C) through the mediation of an if not-A, then not-C conditional. In the literature there is some controversy regarding the pragmatic principle by which $\mathrm{CP}$ is derived, but there seems to be silent agreement about the defeasibility of CP. In this paper we argue that the if not-A, then not-C CP inference is indefeasible with particular conditional utterance types where cancellation contrasts with the additional propositional attitude expressed by those conditional utterance types. The indefeasibility of the if not-A, then not-C CP inference can further be accounted for in terms of the pragmatic principle by which it is derived.
\end{abstract}

(C) 2009 Elsevier B.V. All rights reserved.

Keywords: Implicature; Cancellability; Conditional perfection; Propositional attitude

\section{Introduction}

Content and, to a lesser degree, epistemic or inferential conditionals ${ }^{1}$ regularly invite conditional perfection as a non-monotonic inference or conversational implicature. Conditional perfection (henceforth $\mathrm{CP}$ ) is the natural language tendency to perfect conditionals (if A then $\mathrm{C}$ ) ${ }^{2}$ into their corresponding biconditionals (if and only if A, then C) through the mediation of an if not-A, then not-C conditional. In the literature there is some controversy regarding the pragmatic principle by which CP is derived, but there seems to be silent agreement about the defeasibility of CP. Levinson (2000:117), e.g. collects a whole range of inferences under his rubric of I-inferences, including CP, and subsumes the discussion of the defeasibility of implicatures to a previous chapter. Similarly, Horn (2000), on whose account $\mathrm{CP}$ is considered an instance of R-based strengthening involving the second Maxim of Quantity, does not dilate on the defeasibility of CP either. Neither does Van der Auwera (1997), who considers CP a scalar Q-implicature.

In this paper we summarize and further extend the analysis of Van Canegem-Ardijns and Van Belle (2008) who present evidence for the claim that the inference that if not- $\mathrm{A}$, then not-C correlates with more conditional utterance

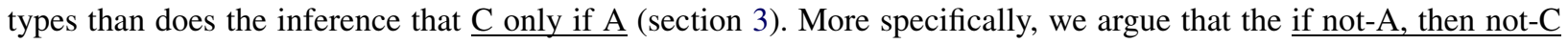

\footnotetext{
* Tel.: +32 163247 81; fax: +3216324767.

E-mail address: ingrid.vancanegem-ardijns@arts.kuleuven.be.

${ }^{1}$ For the distinction between content, epistemic and speech act conditionals, see Sweetser (1990).

${ }^{2}$ The following abbreviations are used throughout the paper: $\mathrm{A}=$ action/event described in the antecedent, $\mathrm{C}=$ action/event described in the consequent, $\mathrm{S}=$ speaker, $\mathrm{H}=$ hearer.
} 


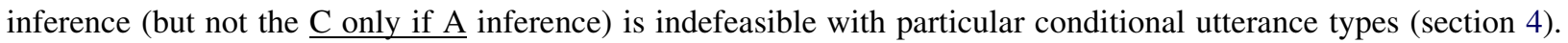
Cancellation of this type of CP inference seems to be viable only to the extent that it does not contrast with the additional propositional attitude expressed by particular conditional utterance types (section 5). We also put forward the hypothesis that the indefeasibility of the if not-A, then not-C CP inference can be accounted for in terms of the pragmatic principle by which it is derived (section 6). But before we proceed, we first spell out why contrary to standard propositional logic we do not consider $\underline{C}$ only if $\mathrm{A}$ and if not- $\mathrm{A}$, then not- $\mathrm{C}$ semantically equivalent (section 2).

\section{2. $\underline{C}$ only if A versus if not-A then not-C}

In the literature CP is associated both with the move from (1a) to (1b) and with that of (1a) to (1b'). It is said that the utterance of (1a) invites the inference (indicated by ' $\sim>$ ') of (1b) and/or (1b'), thus conveying the utterance meaning (indicated by ' $\gg$ ') of (1c).

(1) a. If you mow the lawn, I'll give you five euros.

b. $\quad \sim>$ If you do not mow the lawn, I will not give you five euros.

b'. $\quad \sim>$ Only if you mow the lawn, will I give you five euros.

c. $\gg$ If and only if you mow the lawn, will I give you five euros.

In standard propositional logic (1b) and (1b') are usually considered equivalent. More specifically, many logicians treat 'only if' as a converse of 'if', such that $\underline{\mathrm{C} \text { only if } \mathrm{A}}$ (1b') is equivalent to if $\underline{\mathrm{C} \text {, then } \mathrm{A}}$ (1b').

(1) b". If I will give you five euros, you mow the lawn.

(1b') excludes the possibility that I will give you five euros in circumstances other than ones in which you mow the lawn: mowing the lawn is a necessary condition for my giving you five euros. Now, to say that A is a necessary condition for C is just another way of saying, it is argued by e.g. Copi and Cohen (1998:363-364), that if C holds, A should hold as well. So (1b') can equally well be stated by (1b"), which, by contraposition, is equivalent to (1b).

But on the principle that logical forms should preserve aboutness (Atlas, 1993:304), the alleged equivalence of $\mathrm{C}$ only if $\mathrm{A}$ and if $\mathrm{C}$, then $\mathrm{A}$ is problematic. Whereas 'if' is "ordinarily a sign of the antecedent, the attachment of 'only' reverses it; 'only if' is a sign of the consequent" (McCawley, 1993:81). In (1), but also in (2), for instance, it matters which clause is the antecedent and which the consequent, because 'only if' (2a) reverses the temporal/causal relations expressed by the sentence with 'if' (2b).

(2) a. My pulse goes above 100 only if I do heavy exercise. (McCawley, 1993:82)

b. If my pulse goes above 100 , I do heavy exercise.

c. If I don't do heavy exercise, my pulse doesn't go above 100 .

'Only if' in (2a) and 'if' in (2b) differ sharply in direction of conditionship (Lycan, 2001:38). The condition in (2a) is that I do heavy exercise, while the condition in $(2 b)$ is the condition that my pulse goes above 100 . The latter thus seems to suggest that my pulse going above 100 precedes (and brings about) my doing heavy exercise. The paraphrase in (2c), by contrast, preserves the temporal/causal relations expressed by (2a) in hypothesizing my not doing heavy exercise and suggesting something that will not be triggered by that event. So, McCawley (1981:50) concludes that $\mathrm{C}$ only if $\mathrm{A}$ is better paraphrased by if not-A, then not-C than by if $\mathrm{C}$ then $\mathrm{A}$.

In recent (Horn, 1996; McCawley, 1993; Van Rooij and Schulz, 2007; Von Fintel, 1997; Appiah, 1993) and not so recent studies (Horn, 1969; McCawley, 1981), in which 'only if' is analyzed in a compositional manner as the combination of 'if' and the focussing adverb 'only', it is agreed that the negative contribution expressed by if not-A,

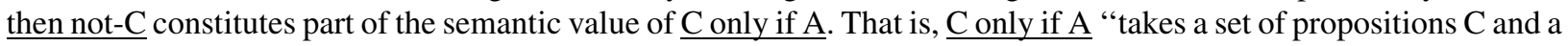
prejacent proposition $p$ and asserts that no proposition in C other than $p$ is true" (Von Fintel, 1997:11), or, alternatively, "that the elements of a set of alternative propositions that differ from the proposition in the scope of 'only' only with respect to the focus value are false" (Van Rooij and Schulz, 2007:194). Intuitively, however, it is quite clear that Conly if $\mathrm{A}$ is not semantically/pragmatically equivalent to if not-A, then not-C, and that in addition to this negative 
component, $\underline{\mathrm{C} \text { only if } \mathrm{A}}$ seems to impose a positive requirement as well, namely $\underline{\mathrm{A} \text { A, then } \mathrm{C}}$ (Von Fintel, 1997:1), as stipulated in (3).

\section{C only if A}

(a) positive contribution: if $\mathrm{A}$ then $\mathrm{C}$

(b) negative contribution: if not-A, then not-C

In line with (3) the utterance in (2a) is typically taken to indicate that if I do heavy exercise, my pulse does in fact go above 100 and that if I do not heavy exercise, my pulse does not go above 100:

(2) My pulse goes above 100 only if I do heavy exercise.

(a) positive contribution: if I do heavy exercise, my pulse goes above 100 .

(b) negative contribution: if I do not do heavy exercise, my pulse does not go above 100 .

The issue of how to account for the positive contribution of 'only if' is a challenging one, though: is it entailed (Van der Auwera, 1985; Atlas, 1991, 1996), presupposed (Horn, 1969; Von Fintel, 1997), pragmatically presupposed/ conventionally implicated (Horn, 1979), or conversationally implicated (McCawley, 1993; Horn, 1992; Van Rooij and Schulz, 2007)? The latter approach takes only the negative contribution to constitute the semantic meaning of $\underline{C}$ only if $\underline{\mathrm{A}}$, which is (negatively) analyzed as if not-A, then not-C, or, alternatively, in terms of the event approach advocated by McCawley (1993:83), as $\mathrm{C}$ in no event other than $\mathrm{A}$. An important observation provided in favour of an asymmetrical analysis of the positive and the negative contribution on which only the latter constitutes part of the semantics of 'only if' concerns the fact that under negation, only the negative contribution is denied (Van Rooij and Schulz, 2007:205).

(2) a'. My pulse goes above 100 not only if I do heavy exercise.

(2a') has the semantic meaning that also in other circumstances my pulse goes above 100, but not that my pulse does not go above 100 if I do heavy exercise. Thus, "the negation behaves as if only the negative contribution but not the positive one is part of the semantic meaning of "only" (Van Rooij and Schulz, 2007:205). Another important observation $^{3}$ in support of an asymmetrical analysis of the positive and the negative contribution relates to their suspension behaviour (Horn, 1996:2; Van Rooij and Schulz, 2007:204; Appiah, 1993:399). Whereas the positive component can be suspended with the help of an epistemic rider 'possible' (2b'), the negative exponent (2c') - "like any true god-fearing entailment" (Horn, 1996:2) - cannot be.

(2) a. My pulse goes above 100 only if I do heavy exercise.

b'. My pulse goes above 100 only if I do heavy exercise and it's possible that even then it does not.

c'. *My pulse goes above 100 only if I do heavy exercise and possibly in some cases in which I don't do heavy exercise.

(2b') seems to indicate that saying $\mathrm{C}$ only if $\mathrm{A}$ does not commit the speaker to assenting if $\mathrm{A}$ then $\mathrm{C}$. The contrast with $\left(2 c^{\prime}\right)$ is "undeniable": cancellation of the negative contribution in (2c') is "as anomalous as any attempt to remove an entailment by the speaker" (Horn, 1996:2).

Horn's (1996) judgements conflict, however, with those of Atlas' (1996) on (2b') and (2c'). In line with his analysis of 'only' in 'only F', Atlas considers (2b') deviant on the epistemic understanding of 'possible' as 'for all I know/believe' "because of the undermining by the second clause of the Felicity Condition on asserting the first" (Atlas, 1996:310). Only on the non-epistemic reading of 'possible' as 'logically possible', does Atlas (1996:300) consider (2b') acceptable.

(2) a. My pulse goes above 100 only if I do heavy exercise.

b'1. * * My pulse goes above 100 only if I do heavy exercise and it's possible that/for all I know even then it does not.

b'2. My pulse goes above 100 only if I do heavy exercise and it's logically possible that even then it does not.

${ }^{3}$ Other objections to an entailment analysis of the positive contribution can be found in Horn (1996:3-4). 
On pursuing Atlas' (1996:300) analysis of the suspension of the positive and negative contribution of 'only' in 'only F' a bit further, he should even find (2c') "much less deviant (in fact marginally acceptable (??)" on the non-epistemic reading of 'possibly' as 'logically possible', though that would probably be pushing things too far.

(2) c'1. ?'My pulse goes above 100 only if I do heavy exercise and it's logically possible that in some cases in which I don't do heavy exercise it does so as well.

On Atlas' account these data refute the asymmetry in acceptability between suspension of the positive and the negative contribution that Horn expects: "there are no felicitous epistemic cancellations of the positive entailment" (Atlas, 1996:310). The positive contribution can only be weakened, but not truly cancelled. In the light of these diagnostics, Atlas casts aside Horn's asymmetric analysis in favour of an analysis which regards the positive contribution not as an implicature, but rather as an entailment of $\underline{\mathrm{C} \text { only if } \mathrm{A}}$.

According to the line pursued by Van Rooij and Schulz (2007:214-215), the asymmetry in acceptability between (2b'1) and (2b'2) can be accounted for in a different way. A sentence of the type $\mathrm{C}$ only if A gives rise to two different pragmatic inferences: one with weak epistemic force that the speaker takes it possible that if $A$ then $C$ and one with strong epistemic force that the speaker knows that if A then $\mathrm{C}$. The epistemic weak inference is hard to cancel, while the epistemic strong inference can be easily suspended. Thus, regarding (2a), the epistemic weak inference that the speaker takes it possible that if he does heavy exercise, his pulse goes above 100 can hardly be cancelled, as illustrated by (2b'1), or, alternatively, by (2b'3). The epistemic strong inference that the speaker knows for a fact that his pulse goes above 100 if he does heavy exercise, by contrast, can easily be cancelled, as illustrated by (2b'2) and (2b'4).

(2) a. My pulse goes above 100 only if I do heavy exercise.

b'3. *My pulse goes above 100 only if I do heavy exercise and (even) if I do heavy exercise, it does not go above 100.

b'4. My pulse goes above 100 only if I do heavy exercise and perhaps even if do heavy exercise, it does not go above 100 .

The general idea is that the epistemic weak inference results from the assumption that the speaker is obeying the Gricean Maxim of Quality and the first Maxim of Quantity, and the epistemic strong inference from the assumption that the speaker is competent on the issue under discussion. The latter assumption is "highly context dependent and therefore easy to cancel", the former assumption on the obedience of the Gricean Maxims is "much more robust and the inferences that follow from this assumption are therefore more difficult to cancel" (Van Rooij and Schulz, 2007:215).

Now, "how, in the face of so many conflicting linguistic intuitions and theoretical claims, shall we go about finding a satisfactory answer" (Atlas, 1991:134)? On the one hand we agree with the objection put forward by Van Rooij and Schulz (2007:205) that the negation of 'only if', exemplified in (2a'), suggests that only the negative but not the positive contribution is part of the semantics of 'only if'. On the other hand, we believe that the problematic nature of the weak epistemic inference cancellation in (2b'1) shows that, though if A then C does not form part of the semantics of $\underline{\mathrm{C} \text { only if } \mathrm{A}}$, what does constitute part of its semantics is that it must at least be possible that if $\mathrm{A}$ then $\mathrm{C}$, or, alternatively, that it cannot be the case that if $A$ then not-C. This means that by saying $\mathrm{C}$ only if $\mathrm{A}$ a speaker entails that if A, it may be the case that C (compare Appiah, 1993:401). To see this, imagine the following context: John is deaf and regardless of whether you shout or not, he will not be able to hear you. In this context if not-A, then not-C (4a) is assertible, but only if A, then C (4b) is not.

(4) a. If you don't shout, John won't hear you.

b. John will hear you only if you shout.

The assertibility of if not-A, then not-C in (4a) is not enough to guarantee the assertibility of $\underline{C}$ only if A in (4b). The reason why the latter is not assertible in this context is that the requirement that if $\mathrm{A}$ it may be the case that $\mathrm{C}$ is violated, for if A (i.e. 'you shout') holds, it cannot be the case that C (i.e. 'John will hear you') holds. ${ }^{4}$ The discrepancy

\footnotetext{
${ }^{4}$ It might be objected that in a context in which John is known to be deaf, the inassertibility of (4b) is due to the falsity of C (i.e. John will hear you). In this view $\mathrm{C}$ only if $\mathrm{A}$ requires that $\mathrm{C}$ be possible. But this requirement is refuted by an utterance like, e.g., 'only if Easter Day falls on a Friday, will John come to the party', in which the falsity of $\mathrm{C}-$ the speaker rules out the possibility that John will come - does not undo the assertibility of the utterance as a whole.
} 
in assertibility of if not- $\mathrm{A}$, then not- $\mathrm{C}$ and $\mathrm{C}$ only if $\mathrm{A}$ is connected with a difference in what constitutes the conditional assumption of the conditional utterance. The latter takes A (i.e. 'you shout' in $4 \mathrm{~b}$ ) as its conditional assumption and, in addition to the entailment that the not-A cases are not-C cases (i.e. the cases in which you don't shout are cases in which he won't hear you), entails - or suggests by a conversational implicature that can at most be weakened, though not cancelled - that it must at least be possible that the $\mathrm{A}$ cases are $\mathrm{C}$ cases (i.e. it must be possible that the cases in which you shout are cases in which he can hear you). The former, by contrast, takes as its conditional assumption not-A (i.e. 'you don't shout' in 4a) and predicts that the not-A cases are not-C cases (i.e. the cases in which you don't shout are cases in which he won't hear you). Only by cancellable implicature does it suggest anything about the A cases, namely that they are $\mathrm{C}$ cases (i.e. the cases in which you shout are cases in which he can hear you). So, in terms of Rescher (2007:7), the enthymematic bases on which the appropriateness of the conditional utterances rests, differ. $\mathrm{C}$ only if A requires a further "background categorical unconditional fact in virtue of which the conditional obtains" (Rescher, 2007:7) in addition to that required by if not-A, then not-C. If the not-A cases are (generally) not-C cases, the

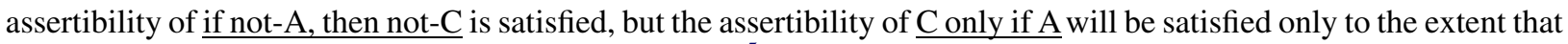
the A cases may be or are in fact (generally) $\mathrm{C}$ cases. $^{5}$

In conclusion, we believe that if not- $\mathrm{A}$, then not- $\mathrm{C}$ and $\mathrm{C}$ only if $\mathrm{A}$ are not semantically equivalent: the former constitutes part of the semantics of the latter, but the latter entails in addition that if $\mathrm{A}$, it may be the case that $\mathrm{C}$. We

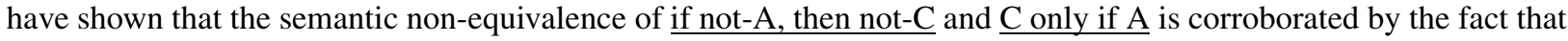
the assertibility of the former is not enough to guarantee the assertibility of the latter: the latter requires that it must at least be possible that if $\mathrm{A}$ is the case, $\mathrm{C}$ is the case as well, whereas the former is assertible in contexts in which this requirement is violated. This discrepancy in assertibility, we have argued, is connected with a difference in what constitutes the conditional assumption of the conditional utterance, i.e. not-A versus A, respectively. Accordingly, the enthymematic bases on which the appropriateness of if not- $\mathrm{A}$, then not-C and $\mathrm{C}$ only if $\mathrm{A}$ rest, differ: in addition to

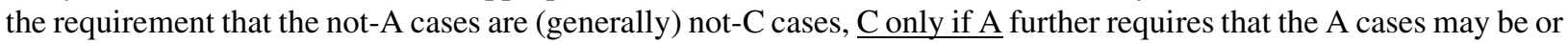
are in fact (generally) $\mathrm{C}$ cases.

\section{If not $\mathrm{A}$, then not $\mathrm{C}$ correlates with greater variety of conditional utterances}

In this section we briefly summarize the findings of Van Canegem-Ardijns and Van Belle (2008) who present evidence for the claim that the if not-A, then not-C inference correlates with a greater variety of speech act or utterance types than does the inference that $\mathrm{C}$ only if $\mathrm{A}$.

The latter inference arises with so-called 'preconditionals' only, i.e. conditional constructions that belong to an utterance type with the following semantic characteristics: [ $\mathrm{S}$ considers $\mathrm{C}$ desirable for $\mathrm{H}$ because $\mathrm{S}$ assumes $\mathrm{H}$ wants $\mathrm{C}$ or $\mathrm{S}$ wants $\mathrm{C} ; \mathrm{S}$ has control over $\mathrm{C}$ ]. In addition, $\mathrm{A}$ is supplied by the speaker as a necessary condition or 'precondition' on the performance of the speech act about $\mathrm{C}$. The $\mathrm{C}$ only if $\mathrm{A}$ inference thus requires that the hearer recognizes that the speaker is in the position to let his performing $\mathrm{C}$ depend solely on the condition stated in A. Typical instances of preconditionals are conditional promises like (1a), where the commitment implied by the speaker's promising that $\mathrm{C}$ will occur, takes effect only if the precondition named by $\mathrm{A}$ is fulfilled.

(1) a. If you mow the lawn, I'll give you five euros.

That is, only if A obtains does the speaker promise to do C. Conversely, the addressee of a conditional promise cannot justifiably say that he has been promised $\mathrm{C}$ without mentioning the condition in A. Other preconditionals are: conditional (non) hearer-directed commitments (e.g. 'if the weather is fine, I'll go for a swim'), conditional cancellations of an order (e.g. 'if you mow the lawn, you do not need to pay me back') and conditional permissions (e.g. 'if you pay your contribution, you may participate in the barbecue').

Besides preconditionals, conditional predictions like 'if you have the operation, you won't die', may also give rise

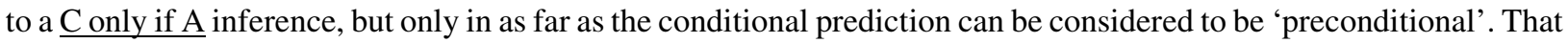

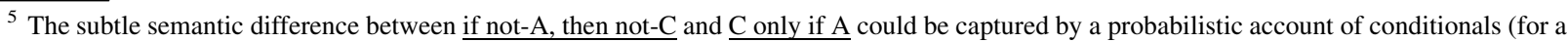
probabilistic account of conditionals, see e.g. Edgington, 2001) as follows: if not-A, then not-C expresses a high probability of not-C given not-A, but leaves aside the probability of $\mathrm{C}$ given $\mathrm{A}$ - it is only by implicature that one can derive high probability of $\mathrm{C}$ given $\mathrm{A}$. $\underline{\mathrm{C} \text { only if } \mathrm{A}}$, on the other hand, expresses not only a high probability of not-C given not-A, but also a (high) probability of $\mathrm{C}$ given $\mathrm{A}$.
} 
is, the speaker needs to be recognized as an expert in case by the hearer (e.g. a doctor to his patient) in that the speaker is in the position to supply A (i.e. the patient having the operation) as a precondition for C (i.e. the patient's not dying). The expertise of the speaker then counts as some sort of control over the outcome of the prediction, given in C. Accordingly, the reason why some conditional utterance types do, while others do not give rise to the $\mathrm{C}$ only if $\mathrm{A}$ inference is that 'only if' requires that the hearer recognizes that the speaker is in the position to let his performing $\mathrm{C}$ (or, in the case of conditional predictions, the outcome presented in $\mathrm{C}$ ) depend solely on the condition stated in A.

The if not-A, then not-C inference, by contrast, correlates ${ }^{6}$ not only with preconditionals, but also with conditional warnings, conditional threats and hypothetical inferential conditionals, i.e. inferential conditionals in which A is not presented as given information grounded in the context of interaction. These conditional utterance types have in common the assumption that the hearer is concerned with the non-realization of $\mathrm{C}$, or, in the case of the hypothetical inferentials, to be eager to know whether or not-C. The point of a conditional warning, for instance, is to inform the addressee of the bad consequence that would result from his doing $\mathrm{A}$, and hence to convince him not to do $\mathrm{A}^{7}$ Accordingly, the addressee may assume that by uttering a conditional warning if $\mathrm{A}$ then $\mathrm{C}$ the speaker means to implicate that if $\mathrm{A}$ does not hold, $\mathrm{C}$ does not hold either as a result (if not- $\mathrm{A}$, then not-C). The assumption that the speaker also means to implicate that $\mathrm{A}$ is the sole circumstance in which $\mathrm{C}$ will occur $(\underline{\mathrm{C} \text { only if } \mathrm{A}})$, by contrast, is not very likely within the context of a conditional warning like (5a) or (6a).

a. If you walk blindly into traffic, you will be hit by a car.

b. $\quad \sim>$ If you don't walk blindly into traffic, you won't be hit by a car.

b'. $\quad ? \sim>$ Only if you walk blindly into traffic, will you be hit by a car. ${ }^{8}$

(6) a. If you touch that wire, you'll get an electric shock.

b. $\quad \sim>$ If you don't touch that wire, you won't get an electric shock.

b'. $\quad ? \sim>$ Only if you touch that wire, will you get an electric shock.

Likewise, if the hypothetical inferential conditional in (7a) is uttered in response to a question like 'Did the plane arrive early?' A is merely hypothetical, and the speaker may be assumed to implicate that if not- $\mathrm{A}$, then not-C on the assumption that the hearer is concerned to know whether or not $C$ holds. But again, there is no reason to assume within the context of the utterance that the speaker meant to implicate that only if she is in the lobby, the plane arrived early.

a. If she is in the lobby, the plane arrived early.

b. $\quad \sim>$ If she is not in the lobby, the plane did not arrive early.

b'. $\quad ? \sim>$ Only if she is in the lobby, did the plane arrive early.

\footnotetext{
${ }^{6}$ In fact, the if not-A, then not-C inference is compatible with an even greater variety of speech act types, e.g. with conditional assertions that assert a causal or temporal relation between A and C, but Van Canegem-Ardijns and Van Belle (2008) argue that if not-A, then not-C arises as a speaker-intended inference only in contexts that display the following characteristics: (i) the speaker may be taken to believe that in situations in which $\mathrm{A}$ is not the case, $\mathrm{C}$ will not be the case as a result; (ii) the speaker may be taken to have the intention to inform the addressee about this belief; (iii) the information that if not-A, then not-C is relevant in the context of the speech act performed by the speaker. The degree of relevance depends on the extent to which the speaker assumes that the addressee is concerned with the non-realization of C. A good reason for such an assumption is that the speaker supposes that the action/event mentioned in $\mathrm{C}$ is (un)desirable for the addressee. With a causal conditional like, e.g., 'if it rains a lot, the ground will become waterlogged' (taken from Declerck and Reed, 2001:45), an if not-A, then not-C interpretation does not invariably arise as an implicature; if, as it most likely is, being meant as a prediction, with the situation in $\mathrm{C}$ predicted to result from the situation in $\mathrm{A}$, the speaker does not mean to implicate anything about the situation in which A is not the case. Also, there is no desire on the part of the addressee that the situation described in $\mathrm{C}$ be realized or not, and, so the information about the non-realization of $\mathrm{C}$ can hardly be considered relevant to the addressee. So, contrary to Dancygier and Sweetser's (2005:39) claim that CP is inevitably linked with predictive conditionals, we believe (and have argued, Van Belle and Van Canegem-Ardijns, 2007:828-830) that predictivity does not count as an explanation for CP, not even of the if not-A, then not-C type. Apart from the fact that some predictive conditionals are incompatible with an if not-A, then not-C reading (e.g. 'if he invites me, I will not go'), we believe that the compatibility of many other predictive conditionals with an if not-A, then not-C reading does not guarantee that the implicature actually arises; it does so only to the extent that that the speaker assumes that the addressee is concerned with the non-realization of $\mathrm{C}$.

${ }^{7}$ Compare with Wierzbicka's (1987:177) analysis of the performative verb to warn: "I think you might do something that would cause something bad to happen to you. I say: [...]. I say this because I want to cause you to be able to cause that bad thing not to happen to you."

${ }^{8}$ We will use the following convention: '* $\sim>$ ' indicates that a particular inference is entirely excluded; '? $\sim$ ' indicates that a particular inference is not entirely excluded, but tends not to arise.
} 
Finally, as to conditional threats ${ }^{9}$ like (8a), they also naturally give rise to an if not-A, then not-C inference, but not to a $\mathrm{C}$ only if $\mathrm{A}$ inference.

(8) a. If you don't pay the rent, I'll throw you out.

b. $\quad \sim>$ If you pay the rent, I won't throw you out.

b'. $\quad ? \sim>$ Only if you don't pay the rent, will I throw you out.

The unacceptability of ( $\left.8 \mathrm{~b}^{\prime}\right)$ is due to the fact that A cannot be regarded as a necessary condition for or precondition on $\mathrm{C}$, for preconditions can be imposed on actions with a positive or desirable orientation only (Athanasiadou and Dirven, 1997:66). Conditional threats are, however, characterized by the negative or undesirable orientation of C [S considers $\mathrm{C}$ undesirable for $\mathrm{H}$ because $\mathrm{S}$ assumes $\mathrm{H}$ does not want $\mathrm{C}]$. Accordingly, the speaker cannot impose the addressee's doing $\mathrm{A}$, i.e. not paying the rent, as a precondition for his doing $\mathrm{C}$, i.e. throwing the addressee out, since one cannot punish somebody on condition that he does something wrong. ${ }^{10}$

To conclude, as an inference, if not- $\mathrm{A}$, then not-C correlates with a greater variety of speech act or utterance types than does $\underline{C}$ only if $\mathrm{A}$. In the latter $\mathrm{A}$ is presented as the sole circumstance in which $\mathrm{C}$ obtains and introduces a precondition on the performance of the speech act associated with $\mathrm{C}$. Hence, $\mathrm{C}$ only if $\mathrm{A}$ arises with preconditionals only, i.e. conditional promises, conditional (non) hearer-directed commitments, conditional cancellations of an order and conditional permissions. The former, by contrast, correlates not only with preconditionals, but also with conditional warnings, conditional threats and hypothetical inferential conditionals.

\section{Cancellability of the inferences $C$ only if $A$ and if not-A then not- $C$}

In the Gricean tradition conversational implicatures are typically differentiated from entailments and semantic implications by the property of defeasibility or cancellability. That is, the former, but not the latter, go through in the absence of information to the contrary, but may be defeated or cancelled in light of new information. Cancellation obtains if the speaker contributes a proposition to the context that is inconsistent with the inference, or if it is evident from the existing context that the inference was not intended. More particularly, in his William James Lectures, Grice observes that a putative conversational implicature $\mathrm{p}$ of an utterance $\mathrm{u}$ may be explicitly cancellable and/or contextually cancellable. It is explicitly cancellable if it is admissible to add to u but not-p or I do not mean to imply that $\mathrm{p}$, without any sense of self-contradiction, and it is contextually cancellable if one can think of a situation in which u would simply not carry the implicature $\mathrm{p}$ (Grice, 1989:39, 44). This property of defeasibility seems to constitute the essential property for modelling inferences labelled as particularized or generalized conversational implicatures (PCIs and GCIs henceforth) by Grice (1989), and often figures as a test to distinguish between what is said and what is implicated. The prevalent view is that any proposition that the speaker is communicating that is distinct from what he says is explicitly cancellable (Grice, 1989:44). ${ }^{11}$ Accordingly, in the literature there is silent agreement about the cancellability of CP: no one seems to question its defeasibility.

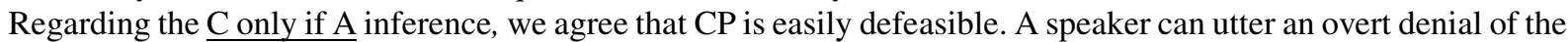
implicature without contradicting himself. The inference that $\mathrm{C}$ only if $\mathrm{A}(9 \mathrm{~b})$, which typically arises with conditional promises (9a), may be cancelled by a clause that supplies a complementary condition but also if B, then $\mathrm{C}$ (9c).
a. If you wash the dishes, I'll help you with your homework.
b. $\quad \sim>$ Only if you wash the dishes, will I help you with your homework.
c. But not only if you wash the dishes, also if you clean the table.

\footnotetext{
${ }^{9}$ Apart from the if not-A, then not-C inference, conditional threats also correlate with a third kind of CP inference distinguished by Van CanegemArdijns and Van Belle (2008), following Fillenbaum (1986), namely only if not-A, then not-C. The intended interpretation of a conditional threat, they argue, is $\mathrm{C}$ unless not-A, which is rhetorically equivalent with $\mathrm{C}$; not-C only if not-A (compare Dancygier, 1998:171-173). The latter paraphrase identifies not-A as the unique circumstance in which $\mathrm{C}$ does not hold, and indicates that the illocutionary force of a conditional threat takes effect irrespective of whether the condition in A obtains. This means that the addressee of a conditional threat can justifiably report on a conditional threat (e.g. 'if you do not pay the rent, I'll throw you out') without mentioning the condition in the antecedent (he threatened to throw me out).

${ }^{10}$ If $\mathrm{S}$ and $\mathrm{H}$ are involved in some kind of agreement, e.g. after a discussion about S's motivations for throwing $\mathrm{H}$ out, $\mathrm{S}$ can posit $\mathrm{A}$ (H's not paying the rent) as a precondition for $\mathrm{C}$ (S's throwing $\mathrm{H}$ out), but in that case $\mathrm{C}$ functions no longer as a genuine threat, but rather as some kind of promise.

${ }^{11}$ Compare with Carston (2002:138), who aligns cancellability with pragmatic inference in general.
} 
As argued with respect to (2a') above, negation of the 'only if' aspect conveys that only the negative contribution (i.e. 'if you do not wash the dishes, I won't help you with your homework') does not hold; the positive contribution (i.e. 'if you wash the dishes, I'll help you with your homework') is preserved under negation. Cancellation of the 'only if' inference does not violate the conditionality of the original speech act; after cancellation A can still be considered a necessary condition for $\mathrm{C}$, alongside with $\mathrm{B}$. This is corroborated by a conversation like (10), where the speaker cancels the hearer's inference that A was meant as the sole precondition for $\mathrm{C}$, while not distancing himself from his original conditional promise.

(10) S: If you wash the dishes, I'll help you with your homework.

H: So if I clean up the room, you won't help me?

S: I will, but still, if you wash the dishes, I'll help you with your homework.

Regarding the if not-A, then not-C inference, however, we do not agree that it is always easily defeasible. Cancellation of this type of $\mathrm{CP}$ involves the use of a clause that rejects $\mathrm{A}$ as a necessary condition for $\mathrm{C}$, but maybe $\mathrm{C}$ anyway. Cancellation does occur with preconditionals like (11a) and (12a). (11a) is a hearer-directed commitment on the part of the speaker that is conditional upon some independent, neither hearer- nor speaker-controlled condition, and (12a) is a non-hearer-directed commitment of the speaker.

(11) a. If the weather clears up again, we'll go for a walk.

b. $\quad \sim>$ If the weather does not clear up, we won't go for a walk.

c. And if the weather does not clear up again, we'll go for a walk as well.

c'. And perhaps if the weather does not clear up again, we'll go for a walk as well.

(12) a. If I can find a new tennis racket, I'll enter the competition.

b. $\quad \sim>$ If I can't find a new tennis racket, I won't enter the competition.

c. And if I can't find a new tennis racket, I'll enter the competition as well.

c'. And perhaps if I can't find a new tennis racket, I'll enter the competition as well.

Both cancellation of the epistemic weak inference that the speaker takes it possible that if not-A, then not-C (c) and cancellation of the epistemic strong inference that the speaker knows that if not-A, then not-C (c') are possible in (11) and (12). An overt denial of the if not-A, then not-C inference gets, however, progressively harder with hearer-directed preconditionals that have a hearer-controlled precondition (9a), with conditional threats (8a) and with conditional warnings (6a).

(9) a. If you wash the dishes, I'll help you with your homework.

d. $\quad \sim>$ If you do not wash the dishes, I won't help you with your homework.

e. ??And if you don't wash the dishes, I'll help you with your homework as well.

e'. ?And perhaps if you don't wash the dishes, I'll help you with your homework as well.

(8) a. If you don't pay the rent, I'll throw you out.

b. $\quad \sim>$ If you pay the rent, I won't throw you out.

c. ??And if you do pay the rent, I'll throw you out as well.

c'. ?And perhaps if you do pay the rent, I'll throw you out as well.

(6) a. If you touch that wire, you'll get an electric shock.

b. $\quad \sim$ If you don't touch that wire, you won't get an electric shock.

c. ??And if you don't touch that wire, you'll get an electric shock as well.

c'. ?And perhaps if you don't touch that wire, you'll get an electric shock as well.

The inferences in (9d), (8b) and (6b) are logically invalid, and yet, cancellation of the epistemic weak inference that the speaker takes it possible that if not-A, then not-C is prohibited. The epistemic strong inference that the speaker knows that if not-A, then not-C is also not easily suspended, witness the limited acceptability of the cancellation with 
'perhaps' in (9e'), (8c') and (6c'). Cancellation of the if not-A, then not-C inference seems to invoke some sort of pragmatic paradox in (9), (8) and (6). The speaker's attempt to get the hearer to wash the dishes in (9), for instance, will be vain on cancellation of the inference in (9d): if the hearer will receive the reward in C irrespective of whether he performs the action named in A, his motivation to do A is seriously challenged. A similar analysis holds for the conditional threat in (8) and the conditional warning in (6). From this it apparently follows that the inference that if not-A, then not-C, if speaker-intended, is not explicitly cancellable with conditionals whereby the speaker performs a hearer-directed speech act and in which A is hearer-controlled. Conditionals of this type have in common an illocutionary goal that can be schematically rendered as the speaker wants the hearer to do/not do A. Cancellation of the inference that if not-A, then not-C is incompatible with this illocutionary goal since the speaker's attempt to get the hearer to do (not-)A, by promising or threatening to do $\mathrm{C}$ in return, or by warning of $\mathrm{C}$, will be vain if $\mathrm{C}$ will happen irrespective of whether A obtains. This also shows from the speaker's paradoxical reaction in a conversation like (13), where he cancels the hearer's inference that if he does not do A, the speaker will not do C, while still holding up his original conditional promise.
S: $\quad$ If you wash the dishes, I'll help you with your homework.
$\mathrm{H}: \quad$ So if I don't, you won't help me?
S: ??I will, but still, if you wash the dishes, I'll help you with your homework.

To conclude, with particular utterance or speech act types the inference that if not-A, then not-C, though logically invalid, can be explicitly defeated only on pain of inviting some sort of pragmatic paradox. More particularly, cancellation of the inference is incompatible with conditional utterances whereby the speaker performs a hearerdirected speech act in which A is hearer-controlled.

\section{Uncancellability of if not-A, then not-C and propositional attitude}

As argued above, cancellation of the CP inference that if not-A, then not-C results in a pragmatic paradox with conditional promises, conditional threats, and conditional warnings. Our claim is that those utterance types express a double propositional attitude, one in addition to the propositional attitude expressed by the non-conditional form of the performed speech act, and that cancellation of the $\mathrm{CP}$ inference that if not- $\mathrm{A}$, then not- $\mathrm{C}$ conflicts with this additional propositional attitude. Below we first discuss the expression of propositional attitudes by promises, threats and warnings. Next, we explain the indefeasibility of the more general type of $\mathrm{CP}$ in terms of the double propositional attitude expressed by conditional promises, threats and warnings.

\subsection{Propositional attitude}

In Searle's (1983) speech act theory the sincerity condition of a speech act specifies the speaker's psychological state with or propositional attitude about the propositional content of the speech act (belief, intention ...). The connection between a speech act and its propositional attitude is regarded as internal, as "the performance of the speech act is necessarily an expression of the corresponding [propositional attitude]" (Searle, 1983:9). The speech act thus commits the speaker to having this particular attitude, possibly without actually having it. Denying the propositional attitude results in a pragmatic, though not logical, inconsistency which arises not from what the speaker said, but from the fact that he said what he said.

The performance of a speech act is eo ipso an expression of the corresponding [propositional attitude]; and, consequently, it is logically odd, though not self-contradictory, to perform the speech act and deny the presence of the corresponding [propositional attitude]. (Searle, 1983:9)

The inconsistency that arises when an expressed propositional attitude is denied is generally known as Moore's paradox (Moore, 1993:207-212). In the illocutionary act of asserting that $p$, for instance, a speaker expresses his belief that $p$. Refuting that belief results in a pragmatic paradox (I assert that $p$ but I do not believe that $p$ ): in everyday language an assertion seems to signal that the speaker believes what he says, though his belief is in no sense a logic or semantic entailment of the propositional contents of his utterance - propositional attitudes, but not entailments, can be denied in reported speech (I said that $p$ though at that time I did not believe that $p$ ). Neither is it an implicature, for it is 
not true that when a speaker says that $p$, he conversationally implicates that he believes that $p$ : it is "not a natural use of language to describe one who has said that $\mathrm{p}$ as having, for example 'indicated', 'suggested' or 'implied' that he believes that $\mathrm{p}$, the natural thing to say is that he has expressed the belief that p" (Grice, 1989:42). The speaker thus "committed himself, in a certain way, to its being the case that he believes that $p$, and while this commitment is not a case of saying that he believes that p, it is bound up, in a special way, with saying that p" (Grice, 1989:42).

By generalization, Moore's paradox arises with speech acts other than assertions as well. Promises and threats, which in speech act theory are analyzed as commissive speech acts whereby the speaker commits himself to carrying out some future course of action, have the sincerity condition that the speaker intends to do what he commits himself to do. Denying that intention is distinctly odd and in some sense self-defeating (I promise to do p, but I do not have the intention to do $\mathrm{p}$, I threaten to do $\mathrm{p}$, but I do not have the intention to do $\mathrm{p}$ ). Warnings, ${ }^{12}$ on the other hand, are analyzed as 'directives' or 'assertives' about some state of affairs. In warning one that $p$, the speaker performs an assertive speech act as he presents the state of affairs that $\mathrm{p}$ as actual, but he typically does so with a view of getting the hearer to do something about it (directive). Conversely, in warning one to do $\mathrm{p}$, the speaker performs a directive speech act as he attempts to get the hearer to carry out p, "while implying that if [the hearer does] not do it, it would be bad for [him] (assertive)" (Searle and Vanderveken, 1987:203). As an assertive speech act about p, warnings have the sincerity condition that the speaker expresses his belief that $\mathrm{p}$; as a directive speech act to do $\mathrm{p}$, they have the sincerity condition that the speaker wants or desires the hearer to do p. Again, denying this belief or desire results in a pragmatic paradox (I warn you that $p$, but I do not believe that $p$ will happen, I warn you to do $p$, but I do not want you to do $p$ ).

\subsection{Uncancellability of if not A, then not $C$}

If in a conditional form, promises and threats do not only express the speaker's intention to perform the future course of action named in $\mathrm{C}$. In a conditional promise or threat the relation between $\mathrm{A}$ and $\mathrm{C}$ is primarily intentional or purposive in nature. The action in C "is really being 'offered' to get something done or not done with regard to [A] on [the hearer's] part" (Fillenbaum, 1986:181). Or, in Akatsuka's (1991:33) terms, a conditional promise roughly conveys the message Do A for me. As a reward, I will do C for you, and a conditional threat conveys the message Do not do A for me. Otherwise, in retaliation, I will do $\mathrm{C}$ to you. Hence, at the motivational level conditional promises and threats also express the speaker's desire that the hearer performs the action or refrains from performing the action named in A (compare Beller, 2002:113). This explains why if a speaker has promised that if A, then C, his performing $\mathrm{C}$ in spite of the hearer's reluctance to perform A, is typically not regarded as a successful accomplishment of the speaker's original promise, but simply as "an irrelevant act, although it might be appreciated as a generous act on the part of the Speaker" (Akatsuka, 1991:36). Conversely, if a hearer consents not to do A in response to the speaker's threat that if A, then C, the speaker's performing $\mathrm{C}$ all the same will not be regarded as a successful accomplishment of the original threat either. The double propositional attitude expressed by conditional warnings easily reads off Searle and Vanderveken's (1987:202-203) analysis of simple warnings summarized above. Conditional warnings are simultaneously assertive and directive, and express both the speaker's belief that there is a causal relation between A and $\mathrm{C}$, and his desire that the hearer does not do A (so as to avoid C).

In brief, the performance of a conditional promise, a conditional threat or a conditional warning is typically understood as the expression of the speaker's desire that the hearer performs or refrains from performing the action in A. Accordingly, a conditional promise is normally intended as an incentive (to do A), giving motivation (in C) to comply with the incentive, and a conditional threat and a conditional warning are normally intended as a prohibition "giving motivation [in C] to comply with the prohibition [in A]" (Comrie, 1986:78). This directive character of conditional promises, conditional threats and conditional warnings is presumably their primary illocutionary goal, which is reflected in Fillenbaum's (1986:179) use of the terms 'conditional inducements' and 'conditional deterrents', respectively.

Conditional promises being normally intended as incentives or inducements, they naturally give rise to the inference that if not-A, then not-C. That is, in response to a conditional promise the hearer typically assumes that if he

\footnotetext{
${ }^{12}$ Wierzbicka (1987:177-179) discusses threats in the same group as warnings. Fillenbaum (1986:186) emphasizes the similarities between threats and warnings: both utterance types allow a disjunctive rephrasing of the form: if $\mathrm{A}$, then $\mathrm{C}=>$ not-A or $\mathrm{C}$, whereas conditional promises correspond to $\underline{A}$ or not-C. Searle and Vanderveken (1987:193) observe the following differences between promises to do $\mathrm{p}$ and threats to do $\mathrm{p}$ : in threats $\mathrm{p}$ is not for the benefit of the hearer, and threatening does not involve obligation.
} 
does not conform to the incentive, he will not receive the promised reward. Alternatively, on hearing a conditional threat or a conditional warning, the hearer will typically assume that if he refrains from doing A and thereby conforms to the prohibition or deterrent, the punishment or bad consequence in $\mathrm{C}$ will not occur. Due to the discourse participants' understanding of what type of additional propositional attitude and corresponding intended speech act is involved, the inference that if not-A, then not-C arises as a "natural consequence" (compare Akatsuka, 1991:36). Quoting Fillenbaum (1986:183), the inference that if not-A, then not-C is not only plausible to make with conditional promises and conditional threats (and, in our view, with conditionals warnings as well), "but not to make it would appear at best foolish, if not perverse".

Interestingly, the speaker's additional propositional attitude, i.e. the expression of desire, and linked with it, the intended incentive or prohibition, is not only explanatory of the ease with which the more general type of CP arises with conditional promises, threats and warnings, it is also explanatory of its indefeasibility. It is indeed pragmatically inconsistent to express one's desire that the hearer performs or refrains from performing the action in A, and to deny the inference that if not-A, then not-C by uttering but (perhaps) if not-A, C as well. In the denial of the inference the motivation behind the intended incentive or prohibition is lost, the expressed desire becomes irrelevant and ineffective, and "the utterance becomes incoherent" (Comrie, 1986:78).

So, our claim is that the indefeasibility of the inference that if not-A, then not-C with conditional promises, threats and warnings can be explained in terms of the double propositional attitude expressed by these conditional utterance types: cancellation of the inference is pragmatically inconsistent with the speaker's expression of desire that the hearer performs or refrains from performing the action named in A. To end with, we wish to show that this contention is further motivated by the defeasibility of the inference that if not-A, then not-C with conditionals like (11) and (12).

(11) a. If the weather clears up again, we'll go for a walk.

b. $\quad \sim>$ If the weather does not clear up, we won't go for a walk.

c. And if the weather does not clear up again, we'll go for a walk as well.

c'. And perhaps if the weather does not clear up again, we'll go for a walk as well.

(12) a. If I can find a new tennis racket, I'll enter the competition.

b. $\quad \sim>$ If I can't find a new tennis racket, I won't enter the competition.

c. And if I can't find a new tennis racket, I'll enter the competition as well.

c'. And perhaps if I can't find a new tennis racket, I'll enter the competition as well.

By uttering the preconditionals in (11a) or (12a) the speaker does not express some kind of desire that the hearer performs or refrains from performing the action named in A. Instead, as argued above, the A in (11a) involves some speaker- and hearer-independent condition, and the A in (12a) involves a speaker- rather than a hearer-controlled condition. Accordingly, no pragmatic inconsistency arises when the speaker denies the inference that if not-A, then $\underline{\text { not-C. }}$.

\section{Uncancellability of if not-A, then not-C and pragmatic principle of derivation}

In the literature there are basically two rival accounts about the pragmatic principle by which $\mathrm{CP}$ is derived. Horn (2000) and Levinson (2000) consider CP as (R-based or, equivalently, I-based) pragmatic strengthening of a sufficient into a necessary and sufficient condition, involving the second Maxim of Quantity (Q2), i.e. not to make one's contribution more informative than is required. On this view the conditional utterance if $\mathrm{A}$, then $\mathrm{C}$ contains extra information in comparison to the stronger assertion of C, and, hence, by Q2 (and the Maxim of Relation/Manner), the stipulated condition $\mathrm{A}$ in if A, then $\mathrm{C}$ must be taken to be informative and relevant, and "what could make such a condition more relevant than its necessity" (Horn, 2000:310). In contrast, Van der Auwera (1997) considers CP a scalar Q-implicature, involving the first Maxim of Quantity (Q1), i.e. to make one's contribution as informative as is required. ${ }^{13}$ On this view, the conditional utterance if $\mathrm{A}$, then $\mathrm{C}$ invokes a set of alternatives, i.e. the additive scale $<$ ((if $\mathrm{A}, \mathrm{C})$ and (if B, C) and (if $\mathrm{D}, \mathrm{C})$ ), ((if $\mathrm{A}, \mathrm{C}$ ) and (if $\mathrm{B}, \mathrm{C})$ ), (if $\mathrm{A}, \mathrm{C})>$, where entailment proceeds downward from the stronger utterance

\footnotetext{
${ }^{13}$ In their exhaustivity analysis of implicatures Van Rooij and Schulz (2004) derive the biconditional reading of 'John will go if Mary will go' as a Q1-implicature.
} 
(if A, C) and (if B, C) and (if D, C) to the weaker utterance (if A, C), and where implicature proceeds upward from the weaker utterance (if $\mathrm{A}, \mathrm{C}$ ) to the negation of the stronger utterance (if $\mathrm{A}, \mathrm{C}$ ) and (if B, C) and (if D, C). Consequently, the assertion of the weaker conditional utterance if A, then $\mathrm{C}$ implicates the inapplicability of the other stronger alternates. Or, in other words, it implicates that there is no other conditional with $\mathrm{C}$ as a consequent.

The two analyses are apparently irreconcilable. Either CP is regarded as exploiting the speaker-based principle of least effort (Q2), or it is regarded as exploiting the hearer-based principle of sufficiency of information $(\mathrm{Q} 1)$. There is a way out, however, if one regards the if not-A, then not-C inference as exploiting the Q2 principle, and the $\mathrm{C}$ only if A inference as exploiting the Q1 principle. Moreover, the indefeasibility of the if not-A, then not-C inference, which contrasts with the defeasibility of the $\underline{\mathrm{C} \text { only if } \mathrm{A}}$ inference, can be explained in terms of the pragmatic principle by which it is derived.

\subsection{Pragmatic principles of derivation of if not-A, then not $C$ and $C$ only if $A$}

In our view, the if not-A, then not-C inference is derived as follows. In harmony with the speaker's assumption that the hearer is concerned with the non-realization of $C$, the speaker's intentionality in uttering if $A$, then $C$ is stereotypically interpreted by the hearer to the more informative reading that its inverse, i.e. if not- $\mathrm{A}$, then not-C, holds as well. The if not-A, then not-C inference is thus considered an R- (or I-) based inference. In contrast to Horn's (2000) analysis, however, the conditional utterance if $\mathrm{A}$, then $\mathrm{C}$ is not regarded as containing extra information which must be taken to be relevant and informative. Rather, in accordance with Levinson's (2000:37) rough characterization of I-inferences, the conditional utterance if $\mathrm{A}$, then $\mathrm{C}$ is regarded as a minimal specification which gets a maximally informative or stereotypical interpretation. The speaker's saying that if $\mathrm{A}$, then $\mathrm{C}$ is then naturally understood as implicating that if not-A, then not-C as well; the inference is taken for granted, given the speaker's assumption of the hearer's concern with the non-realization of C. Of course, the extent to which the speaker may actually be assuming, within the context of his speech act, that the hearer is concerned with the non-realization of $\mathrm{C}$, may vary, and, with it, the salience of the inference that if not-A, then not-C. As argued above, the inference thus most prominently arises with conditional promises, conditional threats and conditional warnings. Due to the strength of the assumption associated with these conditional utterance types, the inference may even arise in cases where it runs counter to other stereotypical assumptions about human transactions. Consider, for instance, (14), which is an atypical conditional promise in that the reward in $\mathrm{C}$ is made conditional upon the hearer not doing a particular chore, and where the readily drawn - inference that 'if you do pick up the garbage, I won't make you a cake' is inconsistent with the commonsensical assumption that a chore is typically rewarded.

If you don't pick up the garbage, I'll make you a cake. (Carston, 1995:222)

Contrary to Carston (1995:222-223) who takes (14) as evidence for the claim 'that neither 'obviousness' nor 'stereotypicality' do the job of accounting for the sort of enrichments exemplified by the so-called I-implicature cases", we do believe that it is stereotypicality which is at stake here. The speaker's assumption about the hearer being concerned about the non-realization of $\mathrm{C}$ is so stereotypically associated or is so 'obvious' with conditional promises, that the inference to this stereotypical interpretation may be drawn even in cases where it runs counter to other stereotypical assumptions.

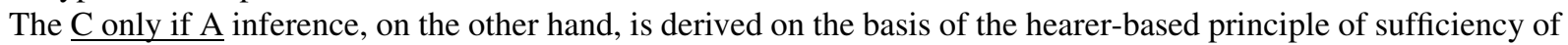
information, according to which the speaker makes his message as informative as is required. Taking into account that the inference arises with conditional utterance types in which the action named in $\mathrm{C}$ is either speaker or hearercontrolled, the speaker's supplying only one condition for $\mathrm{C}$ can naturally be read as there being no other conditions for C. Contrary to Van der Auwera (1997:180), we do not maintain, however, that on the basis of the additive scale $<$ ((if $\mathrm{A}, \mathrm{C}$ ) and (if B, C) and (if D, C)), ((if A, C) and (if B, C)), (if A, C) ${ }^{14}>$, the conditional utterance if A, then C not only implicates that $\mathrm{A}$ is a (ceteris paribus ${ }^{15}$ ) sufficient condition for $\mathrm{C}$, but also Q-implicates that it is not the case that there are other conditions each (ceteris paribus) sufficient for C. As Horn (2000:305) objects, the scale explicitly refers

\footnotetext{
${ }^{14}$ In view of that, it can be argued that the $\mathrm{C}$ only if $\mathrm{A}$ type of $\mathrm{CP}$ does not arise by virtue of the propositional content of the conditional utterance alone, but is licensed by reference to an ensemble of conditional utterances (compare Geurts, manuscript).

${ }^{15}$ See Van der Auwera (1997:179) for a detailed characterization of this ceteris paribus feature.
} 
to "propositional variables for hypothetical states of affairs to be excluded" although these conditions never seem to figure directly in the reasoning involved in CP. As we see it, the hearer is simply reasoning that if there were an antecedent B (other than A) such that if B, then C were true as well, the speaker would have added this conditional to the assertion, hereby appealing to the first Maxim of Quantity: "the speaker of a conditional sentence is cooperative only if the necessary conditions he does not mention are irrelevant or satisfied" (Noordman, 1985:303). ${ }^{16}$

\subsection{Indefeasibility of the if not $A$, then not $C$ as $R$ - or I-based inference}

Significantly, the contention that the if not-A, then not-C inference is derived as an R- or I-based inference and $\mathrm{C}$ only if A inference as a Q-based inference, may be enlightening as well in the light of the indefeasibility of the former inference with particular utterance types. Q-inferences are based on the Q1 Maxim to be as informative as is required, and are "recovered by reference to what else might have been said but was not" (Levinson, 2000:41). They are cancellable given that a speaker may deny that he meant to implicate that what was not said did not hold. Accordingly, a speaker may cancel the $\mathrm{C}$ only if $\mathrm{A}$ inference by denying that he meant to implicate that there is no other antecedent $\mathrm{B}$, other than $\mathrm{A}$, such that if $\mathrm{B}$, then $\mathrm{C}$ is true.

I-inferences, on the other hand, are based on the Q2 Maxim and exploit "only what there is in the sentence: [they are] an inference to a stereotype and as such [are] not so easily cancellable" (Jaszczolt, 2006). We would like to add to this provisory - observation that the more stereotypical or 'default' the inference, the harder it is to cancel it. On this view the extent to which the speaker may be assuming that the hearer is concerned with the non-realization of $\mathrm{C}$ does not only determine the salience with which the general type of CP arises, but also the difficulty with which it can be cancelled.

To finish off, our idea that the defeasibility of the inference that if not-A, then not-C correlates with the 'defaultness' or strength by which it is derived with particular utterance types, seems to be further corroborated by the observation that explicit cancellation of the epistemic weak inference that the speaker takes it possible that if not-A, then not-C (c) and cancellation of the epistemic strong inference that the speaker knows that if not-A, then not-C (c') is acceptable in cases like (15).
a. (Ask him politely to leave the store.) Call the police if he doesn't leave.
b. $\quad$ ?? $>$ 'If he leaves, don't call the police.'
c. And if he leaves, call the police as well.
c'. And perhaps if he leaves, call the police as well.

Given that in (15) the speaker - normally - does not assume that the hearer desires to call the police, he typically does not mean to supply information about the circumstances in which $\mathrm{C}$ does not hold, and, thus, does not mean to implicate that if not-A, then not-C. That is, the conditional utterance is not regarded as a minimal specification which needs to get a maximally informative or stereotypical interpretation according to which its inverse holds as well. Accordingly, the compatibility of an if not-A, then not-C reading with the utterance in (15a) does not guarantee that it actually arises as a relevant implicature (see also note 6) - the irrelevance of the implicature is rendered by the double question marks in (15b). So, in contrast to the conditional promise in (9), the conditional threat in (8) and the conditional warning in (6), the conditional utterance in (15) does not stereotypically give rise to the inference that if not-A, then not-C.

(9) a. If you wash the dishes, I'll help you with your homework.

d. $\quad \sim>$ If you do not wash the dishes, I won't help you with your homework.

e. ??And if you don't wash the dishes, I'll help you with your homework as well.

e'. ?And perhaps if you don't wash the dishes, I'll help you with your homework as well.

(8) a. If you don't pay the rent, I'll throw you out.

b. $\quad \sim>$ If you pay the rent, I won't throw you out.

c. ??And if you do pay the rent, I'll throw you out as well.

c'. ?And perhaps if you do pay the rent, I'll throw you out as well.

\footnotetext{
${ }^{16}$ Or, as von Fintel (2000:13) puts it: "the reason why the speaker would have added such a conditional to the one actually asserted is that the conjunction would have been a statement that should have been asserted (because it gives more of the required information)".
} 
(6) a. If you touch that wire, you'll get an electric shock.

b. $\quad \sim>$ If you don't touch that wire, you won't get an electric shock.

c. ??And if you don't touch that wire, you'll get an electric shock as well.

c'. ?And perhaps if you don't touch that wire, you'll get an electric shock as well.

The easiness with which the inference can be cancelled in (15) and the difficulty with which it can be cancelled in (9), (8) and (6) then seems to be related to the degree of salience by which the implicature arises in the first place.

\section{Conclusion}

The aim of this paper was to chart and explain the constraints on the defeasibility of the CP inference that if not-A, then not-C. We argued that cancellation of the $\mathrm{CP}$ inference that if not-A, then not-C results in a pragmatic paradox with conditional promises, conditional threats and conditional warnings, due to the expression of an additional propositional attitude expressed by those conditional utterance types. In uttering a conditional promise, a conditional threat or a conditional warning the speaker expresses his desire that the hearer performs or refrains from performing the action named in A. By denying the general CP inference, the speaker withdraws the hearer's motivation to comply with the speaker's intended incentive or prohibition, and, hence, the expressed desire becomes irrelevant at most. Further, we have shown that if not-A, then not-C inference should be set apart from the $\mathrm{C}$ only if A inference, as it seems to be derived from another pragmatic principle. In our view, the former exploits the Q2 Maxim, and the latter the Q1 Maxim. This means that the if not-A, then not-C inference is an I-based inference to stereotype, which may also be explanatory of its indefeasibility with particular conditional utterance types.

\section{Acknowledgements}

This research was funded by the K.U. Leuven Research Fund. I would like to thank William Van Belle for his helpful suggestions and for proof reading the article. I would also like to thank the two anonymous referees for their helpful comments.

\section{References}

Akatsuka, Noriko, 1991. Dracula conditionals in discourse. In: Georgopoulos, C., Ishihara, R. (Eds.), Interdisciplinary Approaches to Language: Essays in Honor of S.-Y. Kuroda. Kluwer Academic Publishers, Dordrecht/Boston/London, pp. 25-37.

Appiah, K. Anthony, 1993. Only-ifs. Philosophical Perspectives 7, 397-410.

Athanasiadou, Angeliki, Dirven, René, 1997. Conditionality, hypotheticality, counterfactuality. In: Athanasiadou, A., Dirven, R. (Eds.), On Conditionals Again. John Benjamins, Amsterdam/Philadelphia, pp. 61-96.

Atlas, Jay David, 1991. Topic/comment, presupposition, logical form and focus stress implicatures: the case of focal particles only and also. Journal of Semantics 8, 127-147.

Atlas, Jay David, 1993. The importance of being 'only': testing the neo-Gricean versus neo-entailment paradigms. Journal of Semantics 10, 301318.

Atlas, Jay David, 1996. 'Only' noun phrases, pseudo-negative generalized quantifiers, negative polarity items, and monotonicity. Journal of Semantics 13, 265-328.

Beller, Sieghard, 2002. Conditional promises and threats - cognition and emotion. In: Gray, W.D., Schunn, C.D. (Eds.), Proceedings of the Twentyfourth Annual Conference of the Cognitive Science Society, Lawrence Erlbaum, Mahwah, NJ, pp. 113-118.

Carston, Robyn, 1995. Quantity maxims and generalised implicature. Lingua 96, 213-244.

Carston, Robyn, 2002. Thoughts and Utterances: The Pragmatics of Explicit Communication. Blackwell, Oxford.

Comrie, Bernard, 1986. Conditionals: a typology. In: Traugott, E. Closs, ter Meulen, A., Snitzer Reilly, J., Ferguson, C.A. (Eds.), On Conditionals. University Press, Cambridge, pp. 77-99.

Copi, Irving M., Cohen, Carl, 1998. Introduction to Logic, 10th edition. Prentice Hall, Upper Saddle River, NJ.

Dancygier, Barbara, 1998. Conditionals and Prediction. Time, Knowledge, and Causation in Conditional Constructions. Cambridge University Press, Cambridge.

Dancygier, Barbara, Sweetser, Eve, 2005. Mental Spaces in Grammar: Conditional Constructions. University Press, Cambridge, Cambridge studies in linguistics.

Declerck, Renaat, Reed, Susan, 2001. Conditionals. A Comprehensive Empirical Analysis. Mouton de Gruyter, Berlin/New York.

Edgington, Dorothy, 2001. Conditionals. In: Goble, L. (Ed.), The Blackwell Guide to Philosophical Logic. Blackwell, Oxford, pp. $385-414$.

Fillenbaum, Samuel, 1986. The use of conditionals in inducements and deterrents. In: Traugott, E. Closs, ter Meulen, A., Snitzer Reilly, J.,Ferguson, C.A. (Eds.), On Conditionals. University Press, Cambridge, pp. 179-195. 
Geurts, Bart. Implicatures without propositions, manuscript. Submitted to: http://semanticsarchive.net/Archive/GFhZWU2N/nopropositions.pdf. Grice, H. Paul, 1989. Studies in the way of words. Harvard University Press, Cambridge, Mass.

Horn, Laurence R., 1969. A presuppositional analysis of only and even. CLS Papers from Chicago Linguistic Society 5, 97-108.

Horn, Laurence R., 1979. Only, even, and Conventional Implicature. LSA Paper, Los Angeles.

Horn, Laurence R., 1992. The said and the unsaid. In: Barker, C., Dowty, D. (Eds.), SALT II: Proceedings of the Second Conference on Semantics and Linguistic Theory, Ohio State University, Department of Linguistics, pp. 163-192.

Horn, Laurence R., 1996. Exclusive company: only and the dynamics of vertical inference. Journal of Semantics 13, 1-40.

Horn, Laurence R., 2000. From IF to IFF: conditional perfection as pragmatic strengthening. Journal of Pragmatics 32, 289-326.

Jaszczolt, Katarzyna M., 2006. Defaults in semantics and pragmatics. In: Standard encyclopedia of philosophy. http://plato.stanford.edu/entries/ defaults-semantics-pragmatics/.

Levinson, Stephen C., 2000. Presumptive Meanings. The Theory of Generalized Conversational Implicature. MIT, Cambridge, Massachusetts/ London.

Lycan, William G., 2001. Real Conditionals. Clarendon Press, Oxford.

McCawley, James D., 1981. Everything that Linguists have Always Wanted to Know about Logic but were Ashamed to Ask. University of Chicago Press, Chicago.

McCawley, James D., 1993. Everything that Linguists have Always Wanted to Know about Logic but were Ashamed to Ask, 2nd edition. University of Chicago Press, Chicago.

Moore, George Edward, 1993. Moore's paradox. In: Baldwin, T. (Ed.), G.E. Moore: Selected Writings. Routledge, London, pp. $207-212$.

Noordman, Leo G.M., 1985. On contextual constraints of some conditional conjunctions. In: Hoppenbrouwers, G.A.J., Seuren, P.A.M., Weijters, A.J.M.M. (Eds.), Meaning and the Lexicon: Proceedings of the Second International Colloquium on the Interdisciplinary Study of the Semantics of Natural Language, Foris, Dordrecht, pp. 302-307.

Rescher, Nicholas, 2007. Conditionals. MIT, Cambridge/London.

Searle, John R., 1983. Intentionality. University Press, Cambridge.

Searle, John R., Vanderveken, Daniel, 1987. Foundations of Illocutionary Logic. University Press, Cambridge.

Sweetser, Eve, 1990. From Etymology to Pragmatics. Metaphorical and Cultural Aspects of Semantic Structure. University Press, Cambridge.

Van Belle, William, Van Canegem-Ardijns, Ingrid, 2007. Book review of Dancygier, B., Sweetser, E., 2005. Mental Spaces in Grammar: Conditional Constructions. Cambridge: Cambridge University Press. Linguistics 45, 824-833.

Van Canegem-Ardijns, Ingrid, Van Belle, William, 2008. Conditionals and types of conditional perfection. Journal of Pragmatics 40, 349-376.

Van der Auwera, Johan, 1985. Only if. Logique et analyse 28, 61-74.

Van der Auwera, Johan, 1997. Conditional perfection. In: Athanasiadou, A., Dirven, R. (Eds.), Conditionality, Hypotheticality, Counterfactuality. John Benjamins, Amsterdam/Philadelphia, pp. 169-190.

Van Rooij, Robert, Schulz, Katrin, 2004. Exhaustive interpretation of complex sentences. Journal of Logic, Language and Information 13, $491-519$.

Van Rooij, Robert, Schulz, Katrin, 2007. Only: meaning and implicatures. In: Aloni, M., Butler, A., Dekker, P., Turner, K. (Eds.), Questions in Dynamic Semantics. Elsevier, Amsterdam, Current research in the semantics/pragmatics interface 17.

Von Fintel, Kai, 1997. Bare plurals, bare conditionals, and only. Journal of Semantics 14, 1-56.

Von Fintel, Kai, 2000. Conditional strengthening. A case study in implicature. Unpublished manuscript, http://web.mit.edu/fintel/www/condstrength.pdf.

Wierzbicka, Anna, 1987. English Speech Act Verbs. A Semantic Dictionary. Academic Press, London.

Ingrid Van Canegem-Ardijns is currently a postdoctoral researcher at the Department of Linguistics of the University of Leuven. Her research interests include Dutch syntax and pragmatics and the interface between these two disciplines. 